\title{
Lattice Flavour Physics
}

\section{Nazario Tantalo*}

Rome University "Tor Vergata" and INFN sez. Rome "Tor Vergata"

E-mail: nazario.tantalo@roma2.infn.it

I briefly discuss recent lattice calculations of a selected list of hadronic matrix elements that play a role in flavour physics. I also discuss how the present accuracy on these quantities can eventually be improved by considering effects that have been neglected up to now (isospin breaking, long distance, etc.).

The 2011 Europhysics Conference on High Energy Physics-HEP 2011,

July 21-27, 2011

Grenoble, Rhône-Alpes France

${ }^{*}$ Speaker. 


\section{Introduction}

Thanks to the efforts of several lattice collaborations over the years, there are some phenomenologically relevant hadronic quantities that are presently very well known. These include decay constants of pseudoscalar mesons, form factors of "frequent" semileptonic decay processes, the neutral kaons bag parameter, etc. The theoretical errors on these quantities range from less than $1 \%$ to the few percent level. On the contrary, for quantities as non-leptonic decay rates, flavour singlet meson masses and decay constants, rare semileptonic decays of kaons and beauty mesons etc., lattice predictions are either missing or very rough. This happens because we have learned only recently how to calculate matrix elements involving two pions in external states (but only below the inelastic threshold), because we don't know how to calculate non-leptonic decay rates in general and because it's very difficult to devise efficient numerical strategies to calculate (fermionic) disconnected diagrams and even matrix elements between single hadron states if these are vector mesons or baryons. Here I discuss a very short list ${ }^{1}$ of recent lattice calculations, mainly of "good" quantities (the ones that we know how to calculate), by emphasizing in each case how (and if) better predictions may be obtained.

\section{Leptonic and semileptonic decays of the kaons}

The $K \mapsto \ell v$ and $K \mapsto \pi \ell v$ decays are presumably the best studied processes on the lattice. The corresponding hadronic quantities are $F_{K}$, the leptonic kaon decay constant, and $f_{ \pm}^{K \pi}\left(q^{2}\right)$, the Lorentz invariant form factors parametrizing the semileptonic decay rate. A detailed compilation of recent lattice results for $f_{+}^{K \pi}\left(q^{2}=0\right)$ and $F_{K} / F_{\pi}$, where $F_{\pi}$ is the leptonic decay constant of the pions, has been provided by the FLAG group in ref. [1],

$$
\left[F_{K} / F_{\pi}\right]^{\text {lattice }}=1.193(5), \quad\left[f_{+}^{K \pi}(0)\right]^{\text {lattice }}=0.956(3)(4)
$$

(see also ref. [2] and references therein for recent results not yet included in the FLAG average). Both quantities are known with a $\sim 0.5 \%$ error that combines all sources of lattice uncertainties, i.e. statistics, renormalization, continuum extrapolations, finite size effects, chiral extrapolations, tuning of the quark masses, algorithmic instabilities. In the last few years, the largest source of uncertainty affecting lattice calculations of light pseudoscalar mesons matrix elements was coming from chiral extrapolations. Nowadays, lattice calculations with physical pions and volumes of the order of $6 \mathrm{fm}$ are possible (see for example ref. [3]). On the other hand, lattice QCD algorithms tend to be poorly efficient in sampling long distance degrees of freedom (see for example refs. [4, 5]) and one may wonder whether the errors quoted in eqs. (2.1) are reliable. An answer is provided in ref. [6] where, by assuming the unitarity of the $C K M$ matrix (first row) and by estimating isospin breaking effects by means of chiral perturbation theory [7, 8], experimental measurements combine to give

$$
\begin{array}{ll}
{\left[F_{K} / F_{\pi}\right]^{C K M}=1.1927(59),} & \left(\frac{F_{K} / F_{\pi^{+}}}{F_{K} / F_{\pi}}-1\right)^{\chi p t}=-0.0022(6), \\
{\left[f_{+}^{K \pi}(0)\right]^{C K M}=0.9608(46),} & \left(\frac{f_{+}^{K^{0}} \pi^{0}(0)}{f_{+}^{K^{0} \pi^{-}}(0)}-1\right)^{\chi p t}=0.029(4) .
\end{array}
$$

\footnotetext{
${ }^{1}$ the list is short because, given the time of the talk, this cannot be a comprehensive review and the entries have been selected on the basis of my personal experience.
} 

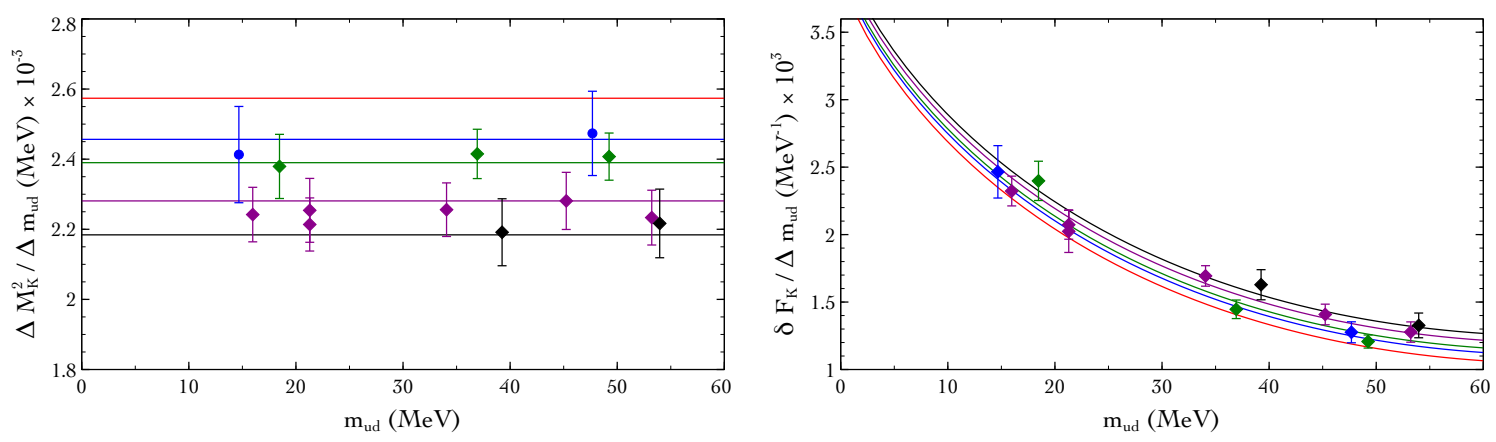

Figure 1: Left panel: lattice results for $\left(M_{K^{0}}^{2}-M_{K^{+}}^{2}\right) /\left(m_{d}-m_{u}\right)$. Right panel: lattice results for $\left(F_{K^{0}}-F_{K^{+}}\right) / F_{K}\left(m_{d}-\right.$ $m_{u}$ ). Results from RM123 collaboration, ref. [9].

Two messages arise from comparing eqs. (2.2) with eqs. (2.1). On the one hand, lattice error estimates may be considered reliable, at least for these quantities. On the other hand, improving the lattice accuracy on $F_{K} / F_{\pi}$ and, in particular, on $f_{+}^{K \pi}(0)$ without taking into account non-perturbatively QCD isospin breaking effects is useless. A first step in this direction has been recently made by the RM123 collaboration in ref. [9] where a method to compute leading QCD isospin breaking effects (the ones coming from $m_{u} \neq m_{d}$ by neglecting electromagnetic interactions) has been devised and applied to several quantities of interest. The results shown in Figure 1 combine to give

$$
\left(\frac{F_{K^{+}} / F_{\pi^{+}}}{F_{K} / F_{\pi}}-1\right)^{\text {lattice }}=-0.0039(3)(2),
$$

a result a bit higher but of the same order of magnitude of the chiral perturbation theory estimate.

\section{Mixing of the neutral kaons}

By reading the previous section one may have got the impression that lattice QCD calculations of matrix elements with single light pseudoscalar mesons in external states can presently be computed with a sub-percent accuracy. Unfortunately this not the case for all the phenomenologically interesting operators and, in the case of four fermion operators, the systematics associated to the renormalization is of the order of a few percent.

Concerning $K^{0}-\bar{K}^{0}$ mixing, on the lattice we know how to calculate the so called bag parameter, $\hat{B}_{K}$. This is given by

$$
\hat{B}_{K}=\left[\frac{\left\langle\bar{K}^{0}\left|H_{W}^{\Delta S=2}\right| K^{0}\right\rangle}{\frac{8}{3} F_{K}^{2} M_{K}^{2}}\right]^{R G I}=\left\{\begin{array}{ll}
0.738(20) & {[1]} \\
0.755(12) & {[10]}
\end{array},\right.
$$

where the first average has been performed by the FLAG group in ref. [1] while the second is the average of the most recent results presented at the last lattice conference, see ref. [10]. The overall relative error of about $2.5 \%$ has to be considered a big achievement of the lattice community and we are at the point where improving the present accuracy on $\hat{B}_{K}$ is useless if we are not able to perform a non-perturbative calculation of the long distance contributions to the $K^{0}-\bar{K}^{0}$ mixing. 
The strength of the previous statement is justified to the light of the observation $[11,12]$ that long distance contributions to $\varepsilon_{K}$ can be parametrized in terms of a correction factor to $\hat{B}_{K}$ that, from a phenomenological estimate, represents a non-perturbative correction of about $6 \%$,

$$
\varepsilon_{K} \propto \kappa_{\varepsilon} \hat{B}_{K}, \quad \kappa_{\varepsilon} \simeq 0.94(2) .
$$

A fully non-perturbative calculation of $\varepsilon_{K}$, and of $\Delta M_{K}$ and $\Gamma_{K}$, is very challenging but it's not completely out of reach. It can be achieved by passing though a direct evaluation of $K \mapsto \pi \pi$ matrix elements in both the $I=0$ and $I=2$ isospin channels or by evaluating euclidean correlators of double insertions of the $\Delta S=1$ weak hamiltonian. Concerning the calculation of $K \mapsto \pi \pi$ amplitudes, huge progresses have been made in the last few years (see for example refs. [10,13]) thanks to the theoretical developments that followed the original proposal of Lellouch and Lüscher [14]. Concerning the double insertion of $H_{W}^{\Delta S=1}$ into kaons two point functions (see ref. [15] for a recent proposal and also refs. [16,9] for related work), one should be able to extract the physical informations from sub-leading exponentials contributing to the euclidean correlators.

\section{A brief excursion in lattice $B$ physics}

Let us consider as a "benchmark quantity" the leptonic decay constant of $B_{(s)}$ mesons. Several lattice calculations of $F_{B_{(s)}}$ have been performed over the years and, at the last lattice conference [17], the following results have been quoted

$$
\left[F_{B}\right]^{n_{f}=2+1}=194(7) \mathrm{MeV}, \quad\left[\frac{F_{B_{s}}}{F_{B}}\right]^{n_{f}=2+1}=1.192(16) .
$$

These figures have been obtained by averaging the latest results of two groups, both using the same gauge ensembles generated with $n_{f}=2+1$ "rooted" staggered quarks, both using effective field theory approaches to heavy quarks on the lattice, Non Relativistic QCD [18] and the FERMILAB approach [19]. Among the results not included in the average are

$$
\left[F_{B}\right]^{n_{f}=2, A L P H A}=172(12) \mathrm{MeV}, \quad\left[F_{B}\right]^{n_{f}=2, E T M C}=195(12) \mathrm{MeV},
$$

obtained, respectively, by the ALPHA collaboration [20] and by the ETMC collaboration [21] with $n_{f}=2$ dynamical flavours of Wilson's like quarks. The ALPHA collaboration approach to heavy quarks on the lattice consists in simulating the lattice discretization of HQET with the inclusion of $1 / m_{h}$ corrections and in performing non-perturbative renormalization and matching by a step-scaling recursive calculation. The ETMC results have been obtained by combining two strategies. The first (standard) strategy consists in performing an interpolation between results obtained with relativistic heavy quarks with masses slightly above the physical value of the charm mass and results obtained in the static limit. The second strategy consists in building suitable ratios of observables evaluated at different heavy quark masses in such a way that the static limit is "exactly" known.

The home message that emerges from the comparison of eqs. (4.1) and (4.2) is that observables associated to $B_{(s)}$ mesons can be presently calculated on the lattice with an accuracy of the order of $5 \%$ or more. This is worse than what we are able to do in the light meson sector because accurate 
lattice simulations of $B_{(s)}$ mesons would require very fine lattice spacings (in order to cope with discretization effects associated with $m_{b}$ ) and large volumes (in order to have small finite volume effects associated with the light quark mass or $\Lambda_{Q C D}$ ). This in turn would require a number of lattice points of the order of $128^{4}$ or more that will be presumably affordable in a few years from now but that is not possible to simulate on presently available supercomputers. The results of eqs. (4.1) and (4.2) tell us that the different strategies devised over the years to cope with such a two-scale problem on the lattice (see also refs. [22]) give consistent results and make us confident on the reliability of the present error estimates. On the other hand, reducing the accuracy at the $1 \%$ level, it will require dedicated efforts and dedicated computer resources. Indeed, having access to computer resources allowing a lattice simulations of $128^{4}$ space-time points, one should decide if to spend this "budget" to get large physical volumes (thus improving the accuracy on light mesons observables) or to get fine lattice spacings and improve the accuracy on heavy mesons observables.

\section{References}

[1] G. Colangelo et al., Eur. Phys. J. C71 (2011) 1695. [arXiv:1011.4408 [hep-lat]].

[2] H. Wittig, Talk given at the XXIX International Symposium on Lattice Field Theory, LATTICE 2011.

[3] S. Durr et al., JHEP 1108 (2011) 148 [arXiv:1011.2711 [hep-lat]].

[4] M. Luscher, PoSLATTICE 2010 (2010) 015 [arXiv:1009.5877 [hep-lat]].

[5] M. Luscher and S. Schaefer, JHEP 1107 (2011) 036 [arXiv:1105.4749 [hep-lat]].

[6] M. Antonelli et al., Eur. Phys. J. C69 (2010) 399-424. [arXiv:1005.2323 [hep-ph]].

[7] A. Kastner and H. Neufeld, Eur. Phys. J. C 57 (2008) 541 [arXiv:0805.2222 [hep-ph]].

[8] V. Cirigliano and H. Neufeld, Phys. Lett. B 700 (2011) 7 [arXiv:1102.0563 [hep-ph]].

[9] G. M. de Divitiis et al., arXiv:1110.6294 [hep-lat].

[10] R.D. Mawhinney, Talk given at the XXIX International Symposium on Lattice Field Theory, LATTICE 2011.

[11] A. J. Buras and D. Guadagnoli, Phys. Rev. D 78 (2008) 033005 [arXiv:0805.3887 [hep-ph]].

[12] J. Laiho, E. Lunghi and R. S. Van de Water, Phys. Rev. D 81 (2010) 034503 [arXiv:0910.2928 [hep-ph]].

[13] T. Blum et al., arXiv:1106.2714 [hep-lat]. T. Blum et al., arXiv:1111.1699 [hep-lat].

[14] L. Lellouch and M. Luscher, Commun. Math. Phys. 219 (2001) 31 [hep-lat/0003023].

[15] N. H. Christ [RBC and UKQCD Collaboration], PoSLATTICE 2010 (2010) 300.

[16] G. Isidori, G. Martinelli and P. Turchetti, Phys. Lett. B 633 (2006) 75 [hep-lat/0506026].

[17] C. Davies, Talk given at the XXIX International Symposium on Lattice Field Theory, LATTICE 2011.

[18] J. Shigemitsu, H. Na, C. Davies, R. Horgan, C. Monahan and P. Lepage, arXiv:1110.5783 [hep-lat].

[19] E.T. Neil, Poster presented at the XXIX International Symposium on Lattice Field Theory, LATTICE 2011.

[20] P. Fritzsch, talk given at the XXIX International Symposium on Lattice Field Theory, LATTICE 2011.

[21] P. Dimopoulos, et al. [ETM Collaboration], arXiv:1107.1441 [hep-lat].

[22] G. M. de Divitiis, E. Molinaro, R. Petronzio and N. Tantalo, Phys. Lett. B 655 (2007) 45 [arXiv:0707.0582 [hep-lat]]. D. Guazzini, R. Sommer and N. Tantalo, JHEP 0801 (2008) 076 [arXiv:0710.2229 [hep-lat]]. G. M. de Divitiis, R. Petronzio and N. Tantalo, Nucl. Phys. B 807 (2009) 373 [arXiv:0807.2944 [hep-lat]]. 